\title{
Carrier dynamics and recombination in silicon doped InAs/GaAs quantum dot solar cells with AlAs cap layers
}

\author{
S. Kondratenko ${ }^{1,2}$, O. Kozak², S. Rozouvan², Yu. I. Mazur ${ }^{3}$, Y. Maidaniuk ${ }^{3}$,

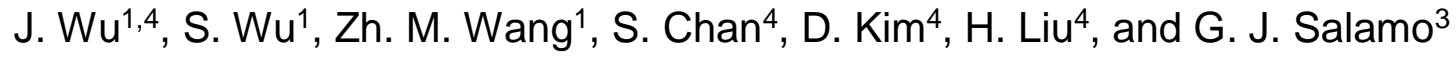 \\ ${ }^{I}$ Institute of Fundamental and Frontier Sciences, University of Electronic Science and Technology of China, \\ Chengdu 610054, P. R. China \\ ${ }^{2}$ Department of Physics, Taras Shevchenko National University of Kyiv, 64 Volodymyrska St., 01601, Kyiv, Ukraine \\ ${ }^{3}$ Institute for Nanoscience and Engineering, University of Arkansas, Fayetteville, Arkansas 72701, United States of \\ America \\ ${ }^{4}$ Department of Electronic and Electrical Engineering, University College London, Torrington Place, London \\ WCIE 7JE, United Kingdom
}

E-mails: kondr@univ.kiev.ua, yumazur@uark.edu

\begin{abstract}
The effects of doping InAs quantum dots (QDs) with Si on charge carrier dynamics and recombination in the InAs/GaAs quantum dot solar cells with AlAs cap layers was investigated. Non-radiative and radiative recombination paths in the doped cells were identified by changes in emission intensity, longwavelength photovoltage (PV) as well as time-resolved PV and photoluminescence (PL) measurements. We find that the reduction of long-wavelength PV and PL with n-doping is due to the electron population of the QD ground states and shrinkage of the depletion layer. The time constants, derived from the timeresolved PV, grow non-monotonically with increasing of the doping density in the QDs due to redistribution of electrostatic potential in the intrinsic region of $\mathrm{p}-\mathrm{i}-\mathrm{n}$ diode and electron population of EL2 defect states of GaAs barriers. We also find that the ground state emission from the InAs QDs decreases with n-doping. The results show that PL traces depends on carrier dynamic in the top QD layers populated partially with electrons from ionized impurities, whereas PV transients were found to be strongly dependent on recombination via QD and defect states located outside the depletion layer. We conclude that the non-radiative recombination of photogenerated electrons and holes via defects is suppressed due to the spatial separation by the local electric fields in and around doped AlAs/InAs QDs, as the potential profile of the intrinsic region is modulated spatially by built-in charges. The interpretation of experimental data suggests limiting mechanisms in the InAs/GaAs quantum dot solar cells operation and sheds light on possible approaches for their further improvement.
\end{abstract}

Keyword: quantum dot solar cells, self-assembled quantum dots, carrier dynamics, recombination. 


\section{Introduction}

InAs/GaAs quantum dot solar cells (QDSCs) have been intensively researched [1,2] in order to achieve an increase in solar power conversion efficiency over the classic Shockley-Queisser limit [3]. A number of approaches have been developed to overcome this limitation. One of them is the intermediate band concept [4],[5] that predicts an enhancement of photocurrent without losses in an open circuit voltage (VOC) when InAs quantum dots (QDs) are incorporated within an intrinsic region of a GaAs p-i-n diode [6]. In such devices, the self-assembled InAs QDs can provide extra optical transitions via quantized energy levels in a spectral region, where the GaAs host material is transparent [7],[8]. However, according to existing reports, InAs/GaAs QDSCs have not yet reached the theoretically predicted photoelectric conversion efficiencies (PCE) ( 19\% to 34\%, depending on operation conditions) [9]. Moreover, the efficiencies of the many reported QDSCs turn out to be lower compared to the GaAs samples without QDs [7],[10]. The two major reasons for such disparity are: strong carrier localization and efficient radiative recombination in InAs QDs, as well as recombination losses via deep defect levels at the InAs/GaAs interface caused by a large lattice mismatch between III-V compounds [11]. In order to improve the photocurrent generation for long wavelengths below the GaAs band gap, numerous approaches have been taken. Among them are methods maximizing the number of stacked QD layers and the QD density [12], methods improving light trapping techniques [5], and those inserting thin barrier layers [13].

Nonetheless, the increase in the PCE due to enhanced infrared (IR) absorption still remains negligible owing to a strong impact on recombination dynamics and charge carrier lifetime of the InAs QDs embedded into GaAs p-i-n junction. The discrete energy levels of the InAs QDs are responsible for the capture and recombination of the charge carriers photogenerated in the GaAs barrier layers [14] and wetting layers (WLs) [15], whereas strain-related defect states provide additional paths for non-radiative recombination [16], deteriorating the SC's performance. As a result, incorporation of QDs into the intrinsic layer of GaAs p-i-n diodes, in addition to IR harvesting, tends to reduce charge carrier's lifetime and deteriorate $V_{\mathrm{OC}}[17]$.

Selective QD doping with Si impurities [18] and barrier layer formation [10,19] have been proposed to decrease recombination loss in the InAs/GaAs QDSCs. In particular, it was found that the n- 
doping of QDs, i.e. negative charging of dots suppresses photoelectron capture and increases the lifetimes of photogenerated electron-hole pairs. Another possibility to suppress thermal coupling between the quantized energy levels of QDs and the conduction band of GaAs is based on formation of a potential barrier around InAs QD by covering it with a AlAs layer that results in carrier confinement potential [20,21]. In such system, the impact of the WL-assisted capture to QDs at room temperatures is reduced due to the relative phase volume of InGaAs WLs reduction, since the In atoms in the WL are substituted by $\mathrm{Al}$ atoms [22]. Secondly, potential barriers around InAs can be modified by selective n-type doping of the QD layer [23]. Such doping has an impact on strain fields, IR photoresponse, and photovoltage (PV) recovery. However, the underlying mechanisms of photogeneration and recombination in the intrinsic region of the doped QDSCs are not yet fully understood.

Therefore, the focus of this work is to analyze the impact of Si doping of AlAs thin capping layers on InAs/GaAs QDSCs performance. In particular, this work will highlight the role of ionized donors and electron population of the QD quantum states in suppression of the electron-hole recombination in the intrinsic region of GaAs p-i-n diodes using time-resolved PV and photoluminescence (PL) data. Understanding the generation-recombination processes and charge carrier dynamics in doped InAs/GaAs QDSC would enable us to assess the origins of the PCE limitations in these devices and to develop a performance improvement strategy.

\section{Experiments}

The GaAs p-i-n diodes with silicon doped InAs/GaAs QDSCs were grown on a n-type GaAs(001) substrates by a solid-source molecular beam epitaxy. QDSC structure consists of: (i) a 200-nm GaAs buffer layer with Si doping density of $1 \times 10^{18} \mathrm{~cm}^{-3}$, (ii) a 30-nm $\mathrm{Al}_{0.35} \mathrm{Ga}_{0.65} \mathrm{As}$ layer field with Si doping density of $1 \times 10^{18} \mathrm{~cm}^{-3}$, (iii) a $1000 \mathrm{~nm} \mathrm{n}$-GaAs intrinsic layer with Si doping density of $1 \times 10^{17} \mathrm{~cm}^{-3}$, (iv) a $250 \mathrm{~nm}$ p-GaAs emitter with Be doping density of $2 \times 10^{18} \mathrm{~cm}^{-3}$, (v) a $100 \mathrm{~nm} \mathrm{p}^{+}$-GaAs layer with Be doping density of $5 \times 10^{18} \mathrm{~cm}^{-3}$, (vi) a $30-\mathrm{nm} \mathrm{Al}_{0.75} \mathrm{Ga}_{0.25} \mathrm{As}$ window layer with Be doping density of 2 $\times 10^{18} \mathrm{~cm}^{-3}$, and (vii) a $50 \mathrm{~nm} \mathrm{GaAs}$ contact layer with Be doping density of $1 \times 10^{19} \mathrm{~cm}^{-3}$. The $\mathrm{Al}_{0.75} \mathrm{Ga}_{0.25} \mathrm{As} / \mathrm{GaAs}$ heterojunction near the illuminated surface was fabricated to reduce an effective recombination velocity near the $\mathrm{p}$-GaAs emitter surface and improve photocurrent due to higher 
separation of charge carriers by the front surface fields [24]. The same motivation was to fabricate a $\mathrm{n}^{+}-$ $\mathrm{Al}_{0.35} \mathrm{Ga}_{0.65} \mathrm{As}$ back surface layer that also improves the open-circuit voltage of GaAs-based solar cells [25]. The intrinsic region of the QDSC contains of 20 stacks of 2.1 monolayers (MLs) InAs QDs separated by undoped $20 \mathrm{~nm}$ GaAs layers. The InAs QD epilayers were covered with 2.0 MLs of AlAs capping layer. The AlAs/InAs QDs were grown in a Stranski-Krastanov mode at a substrate temperature of $\sim 500{ }^{\circ} \mathrm{C}$. The $\mathrm{Si}$ dopants were supplied during the assembling stage of epitaxial growth when impurities are preferentially incorporated into InAs QDs [26]. Therefore, a direct Si-doping of QDs with different densities of dopants $(0,6,12,18$, and 24 dopants per dot $)$ was carried out. A Veeco Nanoscope $\mathrm{V}$ atomic force microscope (AFM) was used to characterize a topology of test samples with an uncapped QD layers. From AFM image of the uncapped epilayer (Figure 1b) is seen that the AlAs/InAs QDs have an average size of $30 \mathrm{~nm}$ in base diameter, $5 \mathrm{~nm}$ in height, and a surface density of about $2.3 \times 10^{10} \mathrm{~cm}^{-2}$. AFM measurements also reveal that doping with Si does not affect the morphology of the QD layers. The initial $5 \mathrm{~nm}$ of the GaAs spacer were grown at $500{ }^{\circ} \mathrm{C}$, then the temperature was increased to $580{ }^{\circ} \mathrm{C}$ for fabrication of final part $(15 \mathrm{~nm})$ of the spacer and reduced back to the growth of next QD layer.

Prior formation of electrodes, the InAs/GaAs QDSCs were cleaned in an ultrasonic bath using acetone and isopropanol for 15 mins by each process, respectively. Finally, to form a good ohmic contact with top p-GaAs surface a grid pattern of gold-zinc alloy (95\% Au, $5 \% \mathrm{Zn})$ was thermally evaporated using a metal mask. As a back contact, a nickel/gold-germanium (88\% Au, 12\% Ge)/nickel/gold (5 $\mathrm{nm} / 150 \mathrm{~nm} / 50 \mathrm{~nm} / 200 \mathrm{~nm}$ thicknesses, respectively) film was deposited on the whole surface of $\mathrm{n}$-GaAs substrate. No anti-reflection coating was fabricated on the top p-GaAs layer. All studied laboratory-type solar cells have an area of $(7 \times 8) \mathrm{cm}^{2}$. Schematic view of a fabricated p-i-n diode with AlAs/InAs QDs is shown in Figure 1.

Photoexcitation and recombination of charge carriers were studied in the Si-doped InAs/GaAs QDSCs with AlAs cap layers by means of PV and PL methods. The cw PL measurements were performed in the temperature range of 10 to $300 \mathrm{~K}$, in a closed-cycle helium cryostat using excitation from $532 \mathrm{~nm}$ line of Nd:YAG laser. The laser spot on the QDSC sample has a diameter of $20 \mu \mathrm{m}$. The PL signal from the sample was collected by mirror optics and dispersed by a monochromator coupled with a liquid nitrogen-cooled InAs photodiode detector array camera (Princeton Instruments, OMAV:512-1.7 $\mathrm{LN})$. 
The time-resolved PL measurements were carried at $10 \mathrm{~K}$ out using a picosecond lifetime measurement system. The PL was excited by $750 \mathrm{~nm}$ line from Ti:sapphire laser with a pulse duration of 2 ps and a repetition rate of $76 \mathrm{MHz}$. A monochromator coupled with a synchroscan streak camera was used to detect PL at different wavelengths. The streak camera with an infrared enhanced S1 cathode provides an overall time resolution of $\sim 15 \mathrm{ps.}$

The measurements of PV spectral dependencies were carried out using a standard infrared spectrometer operating in spectral range $0.5-1.5 \mathrm{eV}$. Unmodulated emission from a $250 \mathrm{~W}$ halogen lamp after passing through monochromator was focused on the QDSC surface. The DC photovoltage spectra and PV transients were measured using a broad range of low noise transimpedance amplifiers. In case of transient measurements, the photoresponse was recorded on a Siglent 70-MHz-bandwidth digital oscilloscope with a pre-amplifier. For transient PV measurements, the QDSCs were excited using an optical pulse generated by a $650-\mathrm{nm}$ and $980-\mathrm{nm}$ laser diodes of pulse width of $\sim 50 \mu \mathrm{s}$, with rise and decay times of $\sim 10 \mathrm{~ns}$. The excitation wavelengths $650 \mathrm{~nm}$ and $980 \mathrm{~nm}$ were set to photoexcite the electron-hole pairs in the p-GaAs top layer or AlAs/InAs QDs, correspondingly. The light pulse intensity was set to induce about $1 \mathrm{mV}$ shift in the barrier height of the $p-i-n$ diodes under illumination. Spectral dependences of the PV were normalized to a constant number of incident photons using supplementary measurements of incident radiation power with a broadband non-selective pyroelectric detector.

\section{Results and discussion}

3.1. Photoluminescence spectroscopy. In order to determine the impact of Si doping on the radiative recombination via QD states, the PL measurements have been carried out for the InAs/GaAs QDSC samples at room temperature. Figure 1c shows how PL spectra of the QDSCs vary with the density of Si impurities in the AlAs/InAs QDs. The undoped sample exhibits a ground-state emission with a peak at $1.099 \mathrm{eV}$ and the high-energy shoulder near $1.165 \mathrm{eV}$ originated from contribution from the excited states. There are two distinctive PL components originating from the interband transitions between the ground states (GSs) and the first excited states of the doped InAs QDs. Electron-holes pairs are excited predominantly in the GaAs barriers by $532 \mathrm{~nm}$ light and relax quickly to the lowest energy states of GaAs barriers and WLs. Thermalized electrons can occupy either ground or excited states of 
QDs, and then recombine radiatively. As the concentration of $\mathrm{Si}$ impurities increases, the relative contribution of the PL from the excited states (high-energy band) also increases due to the changes in the thermal redistribution of carriers to the lowest energy state in QD ensembles. For n-doped QDs, the lowest energy levels of the conduction band are occupied by electrons prior to their optical excitation. Since the number of empty GSs available for photoexcited electrons is reduced with doping, the radiative recombination of electron-hole pairs via ground states of QDs becomes less probable. As a consequence, the relative contribution of the higher excited states increases with n-doping. Interestingly, an increase in the concentration of Si impurities also results in a shifting of the PL to lower energies by several tens of meVs relative to the undoped sample due to Coulomb charging effects [27]. This observation originates from a decrease of electron-hole recombination energy in the presence of additional electrons inside QDs. Despite the same growth conditions, the 6 e/dot sample exhibits a higher redshift compared to the more doped 12 and 18 e/dot samples. We attribute this deviation from the general trend (recombination energy shifts towards higher wavelengths with doping) to the weak variations in the average QD size and composition profile near QD interfaces of different QDSCs when growing the AlAs capping layers and GaAs spacers.
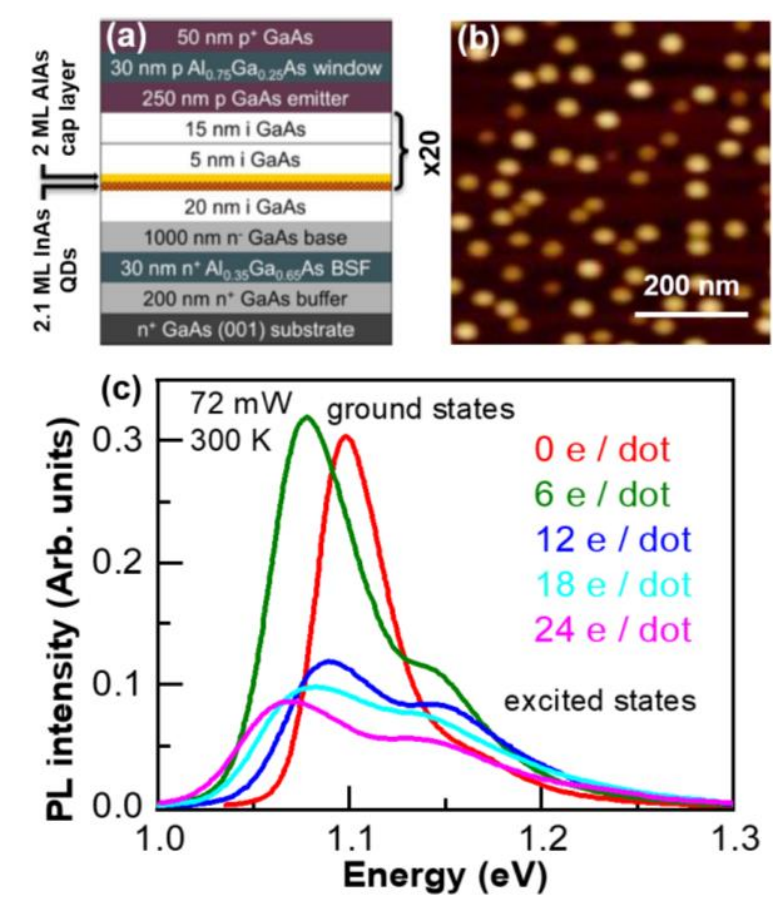

Figure. 1. Structure of the Si-doped InAs/GaAs p-i-n diodes with AlAs/InAs QDs (a), AFM image of the uncapped QD epilayer (b), and PL spectra for the QDSC samples with different doping levels in the QDs measured at $300 \mathrm{~K}$ (c). 
In addition to the observed changes in the shape of the PL spectra indicating substantive electron population of the QD quantum states, the integrated PL non-monotonically changed with doping. The strongest PL intensity was observed for the QDSC with doping density of 6 e/dot due to the repulsive potential profile induced around doped QDs by accumulated electrons, improving the separation of electron-hole pairs [18]. Further increase in the Si doping density leads to a decrease in the integrated PL intensity. This could be attributed to the structural imperfections and defects of QDs, i.e. appearance of additional non-radiative recombination centers responsible for PL quenching.

3.2. $\mathbf{J}-\mathbf{V}$ measurements. The effect of Si doping on non-radiative recombination in the QDSCs was confirmed by analyzing the shape of $J-V$ curves measured at $300 \mathrm{~K}$. Figure 2 shows the representative $J-V$ characteristic for the GaAs p-i-n diode with undoped AlAs/InAs QDs measured under dark conditions. The shape of the curves was analyzed within the framework of the standard twodiode model [28] assuming an impact of the both serial and shunt resistances. The 2/3-Diode software was used for simulations [29]. Generally, the overall current density, $J$, is given by:

$$
J(V)=J_{01}\left(\exp \left(\frac{e\left(V-J R_{S}\right)}{n_{1} k T}\right)-1\right)+J_{02}\left(\exp \left(\frac{e\left(V-J R_{S}\right)}{n_{2} k T}\right)-1\right)+\frac{V-J R_{S}}{R_{S H}}
$$

where $k$ is the Boltzmann's constant, $T$ is the absolute temperature, $R_{\mathrm{S}}$ and $R_{\mathrm{SH}}$ are the series and shunt resistances, $J_{01}$ is saturation current density corresponding to the bulk diffusion with $n_{1}=1$, while $J_{02}$ represents recombination process in the intrinsic region of the GaAs p-i-n diodes [28]. As follows from the simulation, the first term in Eq.(1) was found to be much lower for all samples, while the $n_{2}$ are varied in the range from 2.15 to 2.25 and recombination current dominates for all studied SCs ( $\left.J_{02}>>J_{01}\right)$. This means that the generation-recombination processes via QD states as well as mid-gap states of the GaAs buffers contribute mainly to the saturation diode current. Doping with Si increases $J_{02}$ for concentrations of up to 18 e/dot as ionized donors act as additional Shockley-Reed-Hall (SRH) recombination centers (inset to Fig.2a). Noticeable suppression of the recombination current was achieved with further doping, for 24 e/dot sample only, when the available number of unoccupied confined electron states is reduced significantly. We can explain these results as a consequence of a repulsive Coulomb potential created around the doped InAs QDs. The negatively charged QD can alter 
the electron trajectory in the intrinsic region of the p-i-n diode reducing the probability of their trapping by the QD states and leading to the subsequent recombination with holes injected from p-GaAs [30]. The significant dependence of recombination current on n-doping density in comparison with changes in integrated PL intensity indicates that the non-radiative recombination via defects, rather than radiative recombination, has the dominant effect on charge carrier transport, and will, therefore, limit the performance of the doped QDSCs. To investigate this hypothesis further, we have obtained the J-V dependencies measured under AM1.5G, photovoltage, and external quantum efficiency (EQE) spectra at $300 \mathrm{~K}$. Figure $2 \mathrm{~b}$ shows the J-V dependencies measured under AM1.5G and a light intensity of 100 $\mathrm{mW} / \mathrm{cm}^{2}$. The solar cell parameters such as open-circuit voltage, short-circuit current, fill factor, and photoelectric conversion efficiencies as well as the parameters derived from simulation of the dark J-V curves are presented in the Table S1 of the Supplementary data.
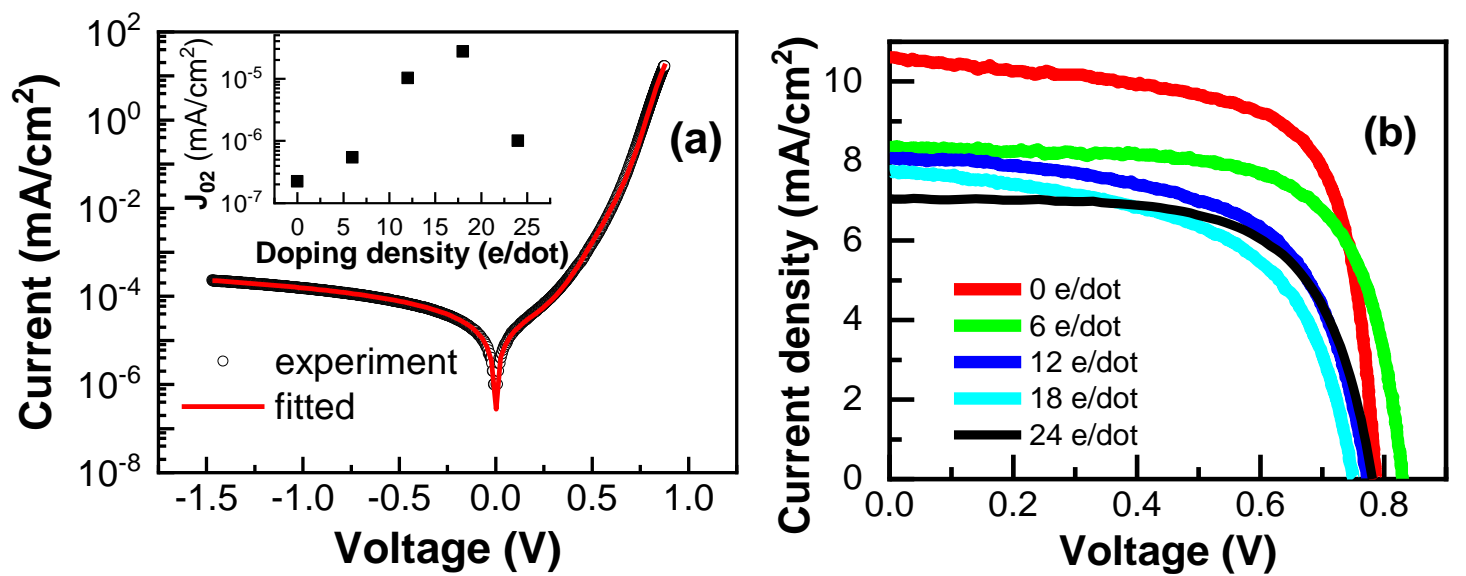

Figure 2. (a) The $J-V$ characteristics for GaAs p-i-n diode with undoped InAs QDs measured under dark conditions at $300 \mathrm{~K}$. Inset shows the dependence of recombination current on doping density. (b) The J-V dependencies measured under AM1.5G and a light intensity of $100 \mathrm{~mW} / \mathrm{cm}^{2}$.

3.3. Photovoltage spectroscopy and external quantum efficiency. As follows from the PV spectra presented in the Fig.3a, besides band-to-band absorption in the GaAs above $1.43 \mathrm{eV}$, we observed absorption in the WL, interband transitions in the InAs QDs, and transition via deep levels in the range from $0.8 \mathrm{eV}$ to $1.43 \mathrm{eV}$. Electron-holes pairs photoexcited in the QDs contribute to the PV spectra in the range 1.0-1.25 eV primary. In particular, two PV bands with maxima at $1.138 \mathrm{eV}$ and 1.216 $\mathrm{eV}$ were observed for the 0 e/dots sample. The low-energy band agrees well with a position of PL peak at $1.099 \mathrm{eV}$ originating from the band-to-band transitions via ground states of QDs, while the PV band near $1.216 \mathrm{eV}$ together shoulder visible on the high-energy side of the PL band are related to transitions via 
first excited states of QDs. In addition to PL spectra, the PV measurements have identified two features in the 1.32-1.43 eV range associated with the interband transitions via higher excited states of QDs (near $1.29 \mathrm{eV}$ ) and $\mathrm{WL}$ (near $1.34 \mathrm{eV}$ ), correspondingly, as well as a contribution of defect-related transition below bandgap-absorption in the InAs QDs $(0.8-1.06 \mathrm{eV})$. The position of band near $1.34 \mathrm{eV}$ agrees well with its predicted energy gap for the AlAs-capped WL. Recently Löbl et.al. [31] have shown that strong emission from the uncapped InGaAs WL observed at $\sim 875 \mathrm{~nm}$, while the WL-related luminescence disappears after capping by AlAs owing to incorporation of AlAs monolayers into InGaAs WL. Somewhat surprisingly that despite an absence of emission from WL in our samples, the PV spectra contain its fingerprints. This is probably because the AlAs barrier layer promotes the spatial separation of electron-hole pairs photogenerated in the WL contributing to PV, whereas the emission becomes suppressed.
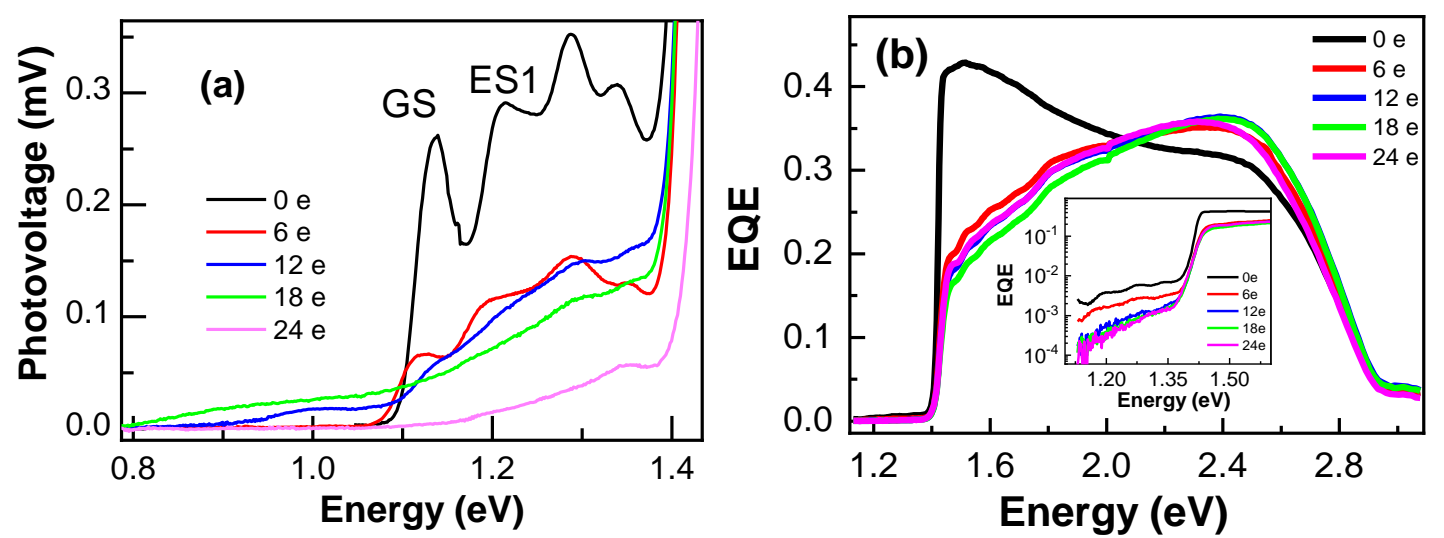

Figure 3. Photovoltage spectra for the QDSC samples with different doping levels in the QDs measured at $300 \mathrm{~K}$ (a); Spectral dependencies of EQE of the QDSCs with different levels of Si doping (b).

A comparison of the PV spectra of QDSCs with different doping density demonstrates that the most significant changes are seen in the region below the energy band gap of GaAs. Figure 3a shows that the introduction of Si dopants leads to the deterioration of IR light harvesting below the energy band gap of GaAs. This decrease of the PV can be explained by a lower probability of interband transitions in the InAs QDs, as the available number of unoccupied confined electron states decreases with doping. For instance, the sample with the highest doping density of $24 \mathrm{e} / \mathrm{dot}$ demonstrates in the 1.06-1.16 eV range the PV a few orders lower than the undoped QDSC. At the same time, changes in PL spectra are not so drastic, indicating the presence of a sufficient number of empty GSs available for radiative recombination of electron-hole pairs photoexcited in the GaAs barriers. This is a clear indication that PL signal results 
from the radiative recombination processes in the top QD layers embedded in the depletion region. To qualitatively illustrate this point, we have simulated the energy band structure of the QDSCs with different levels of Si doping using Nextnano software [32]. As follows from the simulation presented in Figure S1 and Figure S2 of the Supplementary data, there are three energy levels in the conduction band of QD. Therefore, up to six electrons can be confined in a single dot. The AlAs/InAs QDs in the intrinsic region of p-i-n diode is at the nearly constant electric field, whereas doping of QDs results in the significant shrinkage of the depletion region without significant changes in built-in potential. The derived heights of potential barriers in the doped p-i-n diodes agree well with the $V_{\mathrm{OC}}$ values measured under AM1.5G illumination, which varies is the range from $0.786 \mathrm{~V}$ for undoped QDSC to $0.830 \mathrm{~V}$ for a sample with 6 e/dot. Our simulation also demonstrates that at least 16 out of 20 doped QD layers are placed in the region with a nearly flat band and a weak modulation of the electrostatic potential in and around the QDs created by trapped electrons and ionized impurities. Since the Fermi level is close to the position of the ground states of the InAs QDs located outside the depletion layer, they cannot contribute efficiently to the long-wavelength PV below the absorption edge of GaAs due to electron population of the QD quantum states that reduces a long-wavelength light absorption. Furthermore, a limited number of electron-hole pairs that are photogenerated in the more populated QDs outside the depletion region cannot be easily separated to contribute efficiently to the PV signal. Therefore, the main contribution to the lowenergy PV gives the top QD layers placed in the depletion layer.

In addition to the decrease of the long-wavelength PV associated with the interband transitions in the doped QDs, a weak absorption in the low-energy side of the PV spectrum has been observed. This is primarily due to transitions via deep defect levels of EL2 centers that are able to expand the photoresponse of InGaAs/GaAs QD structures to the IR range [33,34]. In the studied QDSCs, defectrelated PV is most pronounced in the $0.8-1.0 \mathrm{eV}$ spectral range for the samples with a doping density of $12 \mathrm{e} / \mathrm{dot}$ and $18 \mathrm{e} / \mathrm{dot}$. It means that direct n-doping of QDs leads to filling of deep defect levels with electrons, which allows for the further photoionization of the EL2 centers contributing to a sub-bandgap (of InAs QDs) PV. Moreover, optical excitation of some defects can also change the potential profile in/around InGaAs QDs and impact SRH recombination of charge carriers in the GaAs barriers [35]. This assumption is consistent with the behavior of the recombination current. The results of $J-V$ analysis demonstrate that the highest saturation current is observed for SCs with the highest long-wavelength 
contribution to PV of EL2 defects. In particular, no transitions via defect states, accompanied by a significant decrease of the saturation current, are observed for a sample with $24 \mathrm{e} / \mathrm{dot}$. This is probably due to compensation of Ga vacancies observed at high concentrations of Si impurity in GaAs, resulting in the decrease of the optically and electrically active defects [36].

Thus, redistribution of potential profile in the intrinsic layer of p-i-n diodes doped with Si results in drastic changes to both recombination and photogeneration processes. To investigate this hypothesis further we have measured the $\mathrm{EQE}$ as a function of quantum energy. The decrease in $\mathrm{EQE}$ with doping in the region below the energy band gap of GaAs shows the same trend as more sensitive low-signal PV measurements (see inset to Fig.3b in comparison with Fig.3a). Having a strong impact on the nonradiative recombination via defect states, doping of Si monotonically decreases the GaAs-related EQE in the range of $h v>1.43 \mathrm{eV}$ (Fig. 3b). The most pronounced changes in the EQE spectra are observed in adsorption near the band-edges of GaAs, when penetration depth of light is comparable with the width of the intrinsic layer. In particular, the EQE of doped sample near $1.6 \mathrm{eV}$ is half the EQE value for both undoped QDSC. Such changes are possibly caused by a decrease in the photogeneration rate of nonequilibrium charge carriers due to shrinkage of the depletion layer observed typically for pin diodes with doped QDs in the intrinsic layer [2].

As a consequence of described changes in the potential profile, a certain part of the electron-hole pairs are photogenerated in depth of the intrinsic layer outside the depletion layer. As a result, their contribution to the EQE in the 1.4-2.2 eV spectral range is suppressed. Photons with higher energies (> $2.2 \mathrm{eV}$ ) are absorbed closer to the illuminated surface in the region with a higher gradient of the electrostatic potential that, in consequence, causes a more efficient spatial separation of photoexcited electrons and holes compared with the undoped sample. This behavior indicates significant redistribution of electrons from ionized donors between QD states and deep defect states that lead to the redistribution of the electric field inside the depletion layer, reduction of photogeneration in the sub-bandgap GaAs IR range, and the decrease in recombination losses via QD states. Despite a substantial improvement in the 2.2-2.8 eV range (relative to undoped sample), the EQE at $300 \mathrm{~K}$ does not exceed $36 \%$, indicating that losses due to a non-radiative recombination via defect states remain substantial. Suppression of recombination losses via states of part of QD layers located in the thinner depletion layer cannot compensate for the reduction of EQE in the 1.4-2.2 eV spectral range. The reason for this fact is that both 
QDs and defects of i-GaAs are effective recombination centers leading to decrease of the both shortcircuit current and PCE from $10.4 \mathrm{~mA} / \mathrm{cm}^{2}$ and $5.6 \%$ (undoped QDSC) to $7.3 \mathrm{~mA} / \mathrm{cm}^{2}$ and $2.9 \%$ (for a sample with 24 e/dot). Taking into account the absence of significant changes in built-in potential and open-circuit voltage due to doping, we believe that PCE can be further improved by proper design of modulation doping profile inside the intrinsic region of a GaAs p-i-n diode focusing on a balance between the population of QDs and redistribution of electrostatic potential. The role of QD layers placed outside the depletion region in carrier dynamics and generation-recombination processes remains unclear.

3.4. Photovoltage and photoluminescence transients. In order to investigate the effects of the ionized impurities of $\mathrm{Si}$ on radiative and non-radiative recombination processes via QD and defect states, the PL and PV transients were studied. Figure 4 presents room temperature PV transients of the QDSCs with different density of the Si impurities using an optical pulse generated by a $650 \mathrm{~nm}$ (a) and a $980 \mathrm{~nm}$ (b) laser diodes with a $50 \mu$ s pulse duration and both leading and trailing edges times of $\sim 10 \mathrm{~ns}$.
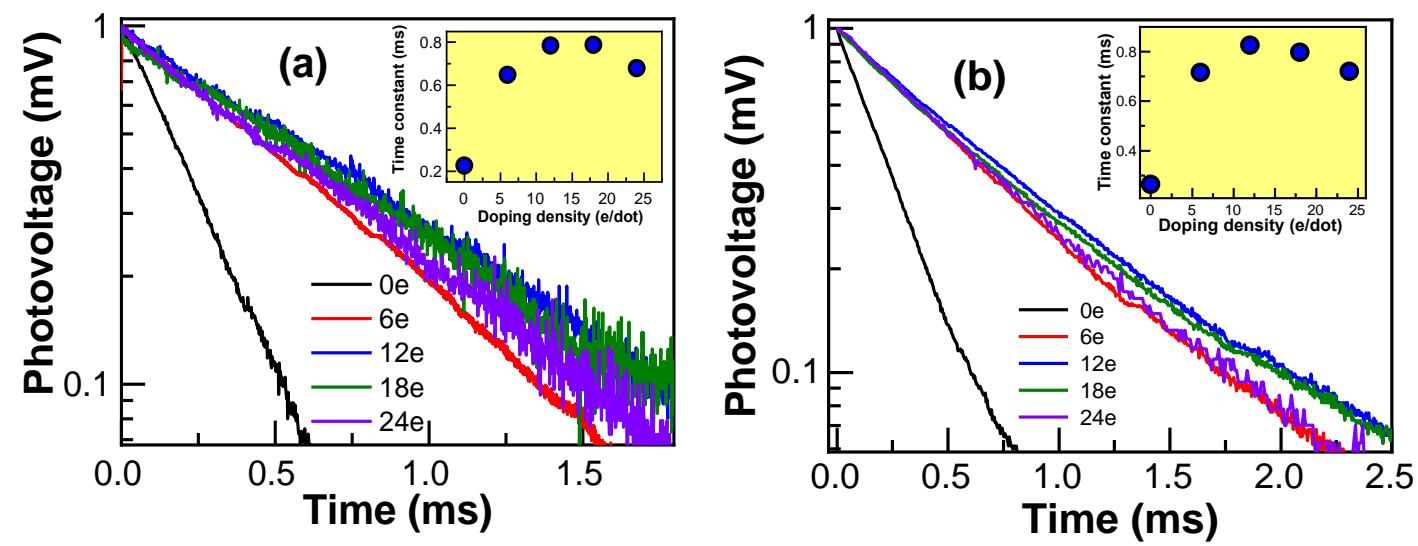

Figure 4. Photovoltage kinetics of the QDSC samples with different doping levels of Si in the AlAs/InAs QDs at $300 \mathrm{~K}$ generated by $650 \mathrm{~nm}$ (a) and $980 \mathrm{~nm}$ (b). The insets show the dependence of PV decay constants on the concentration of Si impurities.

Photovoltage decay traces were found to be exponential with decay time constants, demonstrating evolution depending on the concentration of Si impurities in the InAs QDs (see insets to Fig.4). In comparison with undoped sample, the doped QDSCs have exhibited slower PV decay. The reason is that the deep defect states around negatively charged QDs become less efficient SRH recombination centers due to Coulomb repulsion, which leads to a decrease in the probability of electrons capture and their further recombination with holes. Therefore, $\mathrm{n}$-doping of QDs suppresses the non-radiative recombination of delocalized electrons in the conduction band of GaAs and/or WL states, increasing their lifetimes. 
In order to gain further insight into interband transitions via the states of charged QDs, and to, in particular, clarify the impact of GS's filling on carrier lifetimes, a time-resolved PL (TRPL) measurements were performed. Figure 5a shows the TRPL decay transients of 0 e/dot QDSC taken at $10 \mathrm{~K}$ with excitation by laser pulse $\lambda_{\text {exc }}=750 \mathrm{~nm}$ at different detection wavelengths, $\lambda_{\text {det. }}$ The TRPL traces after termination of excitation were fitted by a single exponential formula: $I_{\mathrm{PL}}(t)=A^{*} \exp \left(-t / \tau_{\mathrm{dec}}\right)$, where $A$ is the pre-exponential constant and $\tau_{\text {dec }}$ is the decay constant. The decay constant exhibits a dependence on detection wavelength that reflects general features observed for continuous-wave PL spectrum recorded at $10 \mathrm{~K}$ (Fig.5b). The same dependencies for doped QDSCs were presented in the Figures S3-S6 of the Supplementary data.
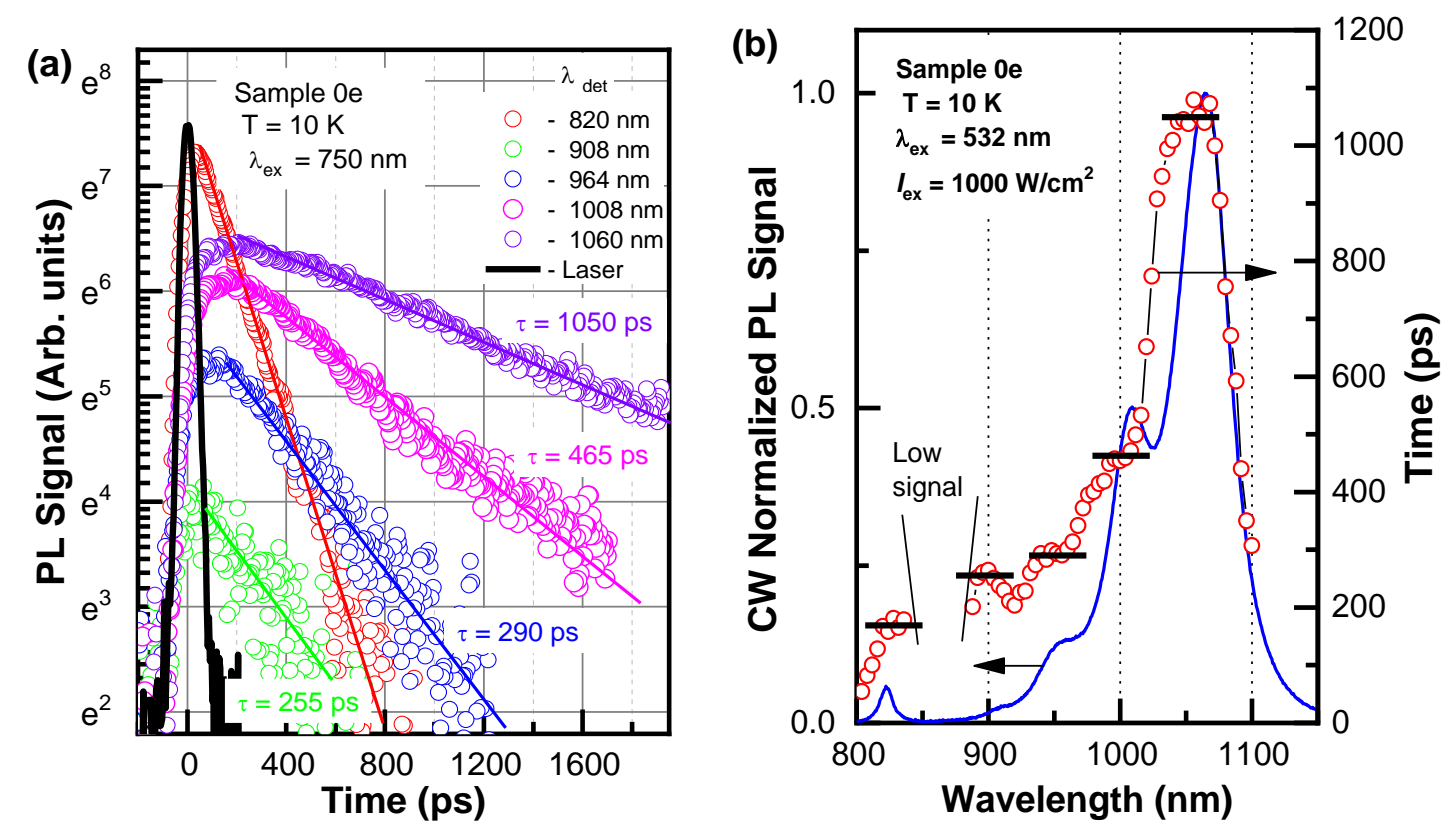

Figure 5. (a) TRPL decay transients with excitation by laser pulse $\lambda_{\mathrm{exc}}=750 \mathrm{~nm}$ at different detection wavelengths and (b) the spectral dependence of decay time constant and continuous-wave PL of 0 e/dot QDSC taken at $10 \mathrm{~K}$.

Figure 6a shows the TRPL decay curves of the QDSC samples with different doping densities of 0, $6,12,18$, and 24 e/dot measured at detection wavelength, corresponding to the recombination between electrons and holes via ground states of the QDs. The increase of n-type doping density from 6 to 24 e/dot decreases the recombination lifetime of carriers at ground states of the QDs from $1084 \pm 40$ to $482 \pm 20$ ps, respectively. The QD ground state carrier lifetimes for samples with different doping density are plotted in the inset to Fig.6a. This observation can be explained as follows. For doped QDs in the depletion layer, the ground states will be randomly occupied by electrons prior to optical excitation of electron-holes pairs into the GaAs barriers. Therefore, ground-state emission from the QDs with an excess of electrons 
depends on rapid capture and relaxation of only the opposite charge carrier (photoexcited holes). Increasing the Si doping density makes the QD ground states more populated; which, in turn, results in a faster relaxation process. At the same time, the InAs QDs located outside the depletion layer cannot contribute to the presented PL traces due to substantive electron filling of the QD ground states, whereas the contribution of excited states or GaAs barriers was possible to detect at shorter wavelengths. In this case, we have observed a significant increase of the time-resolved PL decay constants with Si doping measured at detection wavelengths (see Figures S3-S6 of the Supplementary data). Our study revealed that n-doping of the InAs QDs leads to slowing down of the radiative recombination between electrons and holes via excited states of QDs and bands of GaAs barriers possibly because of decrease the probability for non-radiative recombination pathways inside and around charged QDs.

The assumption was confirmed further by analysis of TRPL measurements at a detection wavelength of $820 \mathrm{~nm}$ corresponding to emission from the GaAs barriers (Fig. 6b). In contradistinction to the undoped sample exhibiting a single exponential decay with $\tau_{\mathrm{dec}}=143 \mathrm{ps}$, the TRPL curves of the doped QDSCs were fitted by a double exponential decay function. Such observation may be due to emission from two regions in the i-GaAs layer with fundamentally different charge carrier dynamics. A slow component, $\tau_{\mathrm{dec} 2}$, is attributed to transport of photoexcited carriers from the GaAs barriers and within WL plane towards the doped QDs in the intrinsic region with the nearly constant electric field. The $\tau_{\mathrm{dec} 2}$ gradually increases with doping from decay constant of 326 ps for $6 e$ sample to 427 ps for $24 \mathrm{e}$ sample (see inset to Fig.6b). Prolongation in the PL decay with doping is due to modulation of the electrostatic potential around charged QDs. At the same time, a shorter time constant $\tau_{\mathrm{dec} 1}$ varies in the range from 44 ps to 78 ps. These values are determined by fast capture from the GaAs spacers between the top QD layers in the shrinking depletion region. The observed variation in $\tau_{\mathrm{dec} 1}$ can be attributed to interplay between a faster electron-hole separation by high electric field in the depletion region and electron population of QD layers prolonging PL relaxation. 

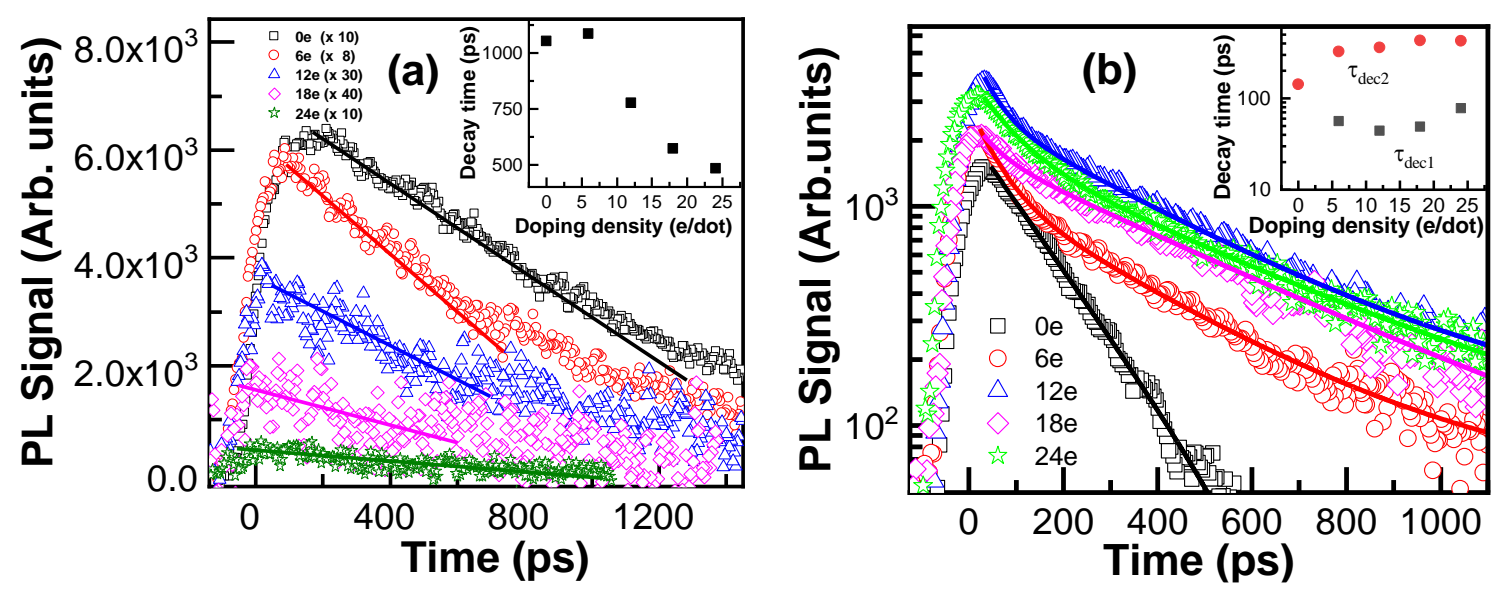

Figure 6. (a) TRPL decay curves of the QDSC samples with different doping densities of $0,6,12,18$, and 24 e/dot measured at detection wavelength, corresponding to the transitions between ground states of the QDs. The inset shows the dependence of decay constant related to GS emission on doping density. (b) TRPL decay curves of the QDSC samples with different doping densities of $0,6,12,18$, and 24 e/dot measured at a detection wavelength of $820 \mathrm{~nm}$, corresponding to the emission from the GaAs barriers. The inset shows the dependence of decay constants derived from fitting by a double exponential decay function $I_{\mathrm{PL}}(t)=A_{1} * \exp \left(-t / \tau_{\operatorname{dec} 1}\right)+A_{2} * \exp \left(-t / \tau_{\mathrm{dec} 2}\right)$, where $A_{1}$ and $A_{2}$ are the pre-exponential constants, $\tau_{\mathrm{dec} 1}$ and $\tau_{\mathrm{dec} 2}$ are the decay constants.

Besides the impact on emission from the doped QDs and GaAs barriers, the application of $\mathrm{Si}$ dopants to the AlAs/InAs QDs significantly decreases the overall recombination losses, thereby prolonging the PV decay. We can conclude that the observed changes in PV decay transients can be explained by appearance of the local electric fields in and around QDs and their impact on non-radiative recombination of electron-holes pairs via point defects into the GaAs barriers. As a consequence, the electron-hole pairs photoexcited in the GaAs barrier layers are separated spatially by built-in charges inside and around QDs leading to the increase of their lifetimes and slowing down an excited state emission in the doped QDs.

Summarizing our discussion, the experimental findings suggest that both radiative and nonradiative recombination depends on the spatial redistribution of electrostatic potential due to the presence of Si impurities in the intrinsic layer of the GaAs p-i-n diodes. In particular, shrinkage of the depletion layer leads to the appearance of two regions with fundamentally different conditions for spatial separation of photoexcited electron-hole pairs, charge carrier dynamics and recombination of the photoexcited electron-hole pairs (Figure 7). Radiative recombination processes take place mainly in the top QD layers located in the depletion region, whereas emission from all other QDs is suppressed due to electron population. Low-energy shift (several tens of $\mathrm{meV}$ ) of the interband transition energy between QD 
electron and hole states together with PL spectra broadening and the decrease of PL decay constant constitute further evidence of the QD negative charging. Such behavior suggests that the losses associated with radiative recombination can be reduced by direct n-doping of InAs QDs that is expected to improve SC performance.
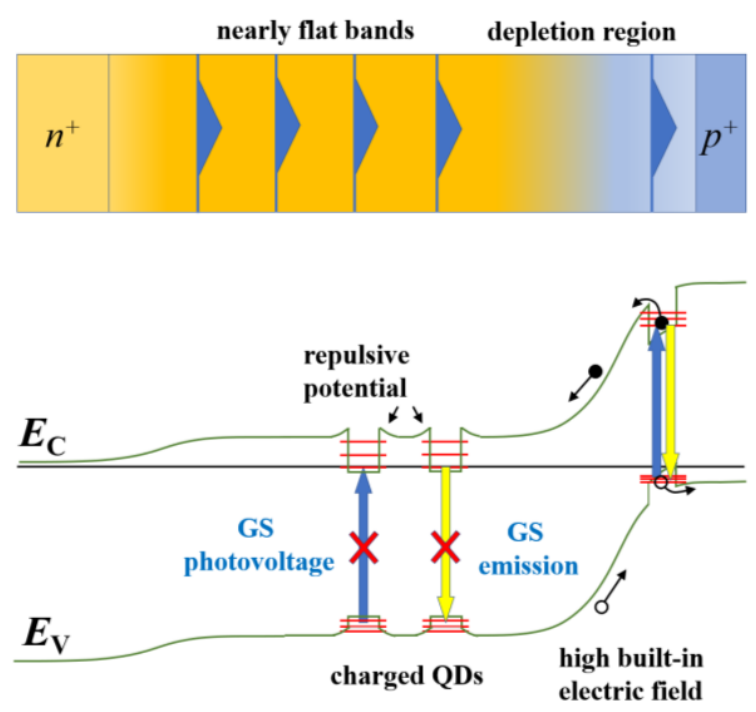

Figure 7. Schematic of the energy band diagram for the doped QDSCs showing two part of $i$-GaAs layer: (i) region with a nearly flat bands and a weak modulation of the electrostatic potential in and around the QDs and (i) the depletion region with top QD layers. To better presentation, 3 layers of 20 are shown only.

On the other hand, the presence of electrons inside the QD reduces the absorption probability, leading to the decrease of the long-wavelength PV. Such behavior suggests that a certain part of the intrinsic layer becomes more transparent for low-energy photons with energies below the bandgap of GaAs (see QD layers outside the depletion region in Fig.7). We have found that together with the reduced IR harvesting in the sub-bandgap region of GaAs, the presence of $\mathrm{Si}$ impurities in the InAs QDs significantly decreases the non-radiative recombination losses, thereby prolonging the PV decay without losses in the open-circuit voltage. In addition, we have observed an increase of PV caused by EL2 defects in the GaAs buffer layer. It means that n-doping also leads to electron populating of deep defect states. This makes further photoionization possible, simultaneously contributing to PV in the $0.8-1.0 \mathrm{eV}$ spectral range. The EL2 defect, related to the As antisite in GaAs, is the double donor with two energy levels near the middle of the bandgap and is the dominant non-radiative recombination center in GaAs. [37] From the general point of view, the efficiency of SRH recombination can be expected to be strongly dependent on the charge state of a defect. Electron population of EL2 results in the reduction of the non-radiative 
recombination rate in the GaAs barriers, leading to the significant dependence of the saturation current on the doping level, as detected by $J-V$ measurements. This study not only points to the significance of the impact of Si doping on both radiative and non-radiative recombination but also shows the significance of the deep levels in GaAs barriers and ionized donors to reduce overall recombination losses in the QDSCs.

\section{Conclusions}

We have demonstrated that the dependence of QDSC performance on Si dopants can be attributed to the shrinkage of the depletion layer and electron population of the InAs QDs and defect states. The experimental findings suggest that both radiative and non-radiative recombination paths depend differently on appearance of two regions in the intrinsic layer of the GaAs p-i-n diodes: one is depletion region and the other is the part of doped i-GaAs layer with a nearly flat band and a weak modulation of the electrostatic potential created by ionized Si impurities. Interband transitions take place mainly in the top QD layers located in the depletion region, whereas absorption and emission via GSs from all other QDs is suppressed due to electron population. We have observed a reduction of long-wavelength PV with n-doping due to the electron population of QDs accompanied by appearance of the contribution from EL2 defects. The long-wavelength PV and EQE were found to be dependent principally on non-radiative recombination via defect states in and around the QDs located outside the depletion layer. The nonradiative recombination of photogenerated electrons and holes via defects is suppressed due to spatial separation by the local electric fields in and around doped AlAs/InAs QDs, as the potential profile of the intrinsic region is modulated spatially by built-in charges increasing carrier lifetime. Opposite trends in dependencies of PV and PL decay (corresponding to GS emission of the QDs) constants on n-doping density as well as a variation of recombination current provides further evidence of a significant impact of electron population of QDs, defects, and ionized donors on the overall recombination losses in the ndoped QDSCs. Such behavior suggests the presence of a high correlation between the carrier dynamics, recombination losses, and redistribution of potential profile in the intrinsic layer of the p-i-n diodes doped with Si. 
The authors acknowledge financial support from the U.S. National Science Foundation (EPSCoR Grant \# OIA1457888).

\section{Notes}

The authors declare no competing financial interest.

\section{ORCID iDs}

S Kondratenko https://orcid.org/0000-0002-4403-7732

S Rozouvan https://orcid.org/0000-0001-8024-6913

\section{References}

[1] J. Wu, Y.F.M. Makableh, R. Vasan, M.O. Manasreh, B. Liang, C.J. Reyner, D.L. Huffaker, Strong interband transitions in InAs quantum dots solar cell, Appl. Phys. Lett. 100 (2012). https://doi.org/10.1063/1.3681360.

[2] F. Cappelluti, M. Gioannini, A. Khalili, Impact of doping on InAs/GaAs quantum-dot solar cells: A numerical study on photovoltaic and photoluminescence behavior, Sol. Energy Mater. Sol. Cells. $157 \quad$ (2016) 209-220. https://doi.org/10.1016/j.solmat.2016.05.049.

[3] W. Shockley, H.J. Queisser, Detailed balance limit of efficiency of p-n junction solar cells, J. Appl. Phys. 32 (1961) 510-519. https://doi.org/10.1063/1.1736034.

[4] A. Luque, A. Martí, Increasing the Efficiency of Ideal Solar Cells by Photon Induced Transitions at Intermediate Levels, Phys. Rev. Lett. 78 (1997) 5014-5017. https://doi.org/10.1103/PhysRevLett.78.5014.

[5] A. Mellor, A. Luque, I. Tobías, A. Martí, The feasibility of high-efficiency InAs/GaAs quantum dot intermediate band solar cells, Sol. Energy Mater. Sol. Cells. 130 (2014) 225233. https://doi.org/10.1016/j.solmat.2014.07.006.

[6] T. Li, R.E. Bartolo, M. Dagenais, Challenges to the concept of an intermediate band in InAs/GaAs quantum dot solar cells, Appl. Phys. Lett. 103 (2013). https://doi.org/10.1063/1.4822322.

[7] D. Guimard, R. Morihara, D. Bordel, K. Tanabe, Y. Wakayama, M. Nishioka, Y. Arakawa, Fabrication of InAs/GaAs quantum dot solar cells with enhanced photocurrent and without degradation of open circuit voltage, Appl. Phys. Lett. 96 (2010). https://doi.org/10.1063/1.3427392.

[8] N.S. Beattie, P. See, G. Zoppi, P.M. Ushasree, M. Duchamp, I. Farrer, D.A. Ritchie, S. Tomić, Quantum Engineering of InAs/GaAs Quantum Dot Based Intermediate Band Solar Cells, 
https://doi.org/10.1021/acsphotonics.7b00673.

[9] S. Tomic, T. Sogabe, Y. Okada, In-plane coupling effect on absorption coefficients ofInAs/GaAs quantum dots arrays for intermediate band solar cell, Prog. Photovoltaics Res. Appl. 23 (2015) 546-558. https://doi.org/10.1002/pip.2455.

[10] P. Lam, S. Hatch, J. Wu, M. Tang, V.G. Dorogan, Y.I. Mazur, G.J. Salamo, I. Ramiro, A. Seeds, H. Liu, Voltage recovery in charged InAs/GaAs quantum dot solar cells, Nano Energy. 6 (2014) 159-166. https://doi.org/10.1016/j.nanoen.2014.03.016.

[11] S.M. Willis, J.A.R. Dimmock, F. Tutu, H.Y. Liu, M.G. Peinado, H.E. Assender, A.A.R. Watt, I.R. Sellers, Defect mediated extraction in InAs/GaAs quantum dot solar cells, Sol. $\begin{array}{llllll}\text { Energy } & \text { Mater. } & \text { Sol. } & \text { Cells. } & 102 & \text { (2012) }\end{array}$ https://doi.org/10.1016/j.solmat.2012.03.010.

[12] W. Rouis, A. Sayari, M. Nouiri, M. Ezzdini, S. Rekaya, L. El Mir, L. Sfaxi, H. Maaref, Characterisation of the GaAs-based intermediate band solar cell with multi-stacked InAs/InGaAs quantum dots, Int. J. Nanotechnol. $12 \quad$ (2015) 584-596. https://doi.org/10.1504/ijnt.2015.068880.

[13] D. Hu, C.C. Mcpheeters, E.T. Yu, D.M. Schaadt, Improvement of performance of inas quantum dot solar cell by inserting thin alas layers, Nanoscale Res. Lett. 6 (2011). https://doi.org/10.1186/1556-276X-6-83.

[14] M. Gioannini, A.P. Cedola, N. Di Santo, F. Bertazzi, F. Cappelluti, Simulation of quantum dot solar cells including carrier intersubband dynamics and transport, IEEE J. Photovoltaics. 3 (2013) 1271-1278. https://doi.org/10.1109/JPHOTOV.2013.2270345.

[15] E. Antolín, A. Martí, C.D. Farmer, P.G. Linares, E. Hernández, A.M. Sánchez, T. Ben, S.I. Molina, C.R. Stanley, A. Luque, Reducing carrier escape in the InAs/GaAs quantum dot intermediate band solar cell, J. Appl. Phys. 108 (2010). https://doi.org/10.1063/1.3468520.

[16] I.S. Han, J.S. Kim, J.O. Kim, S.K. Noh, S.J. Lee, Fabrication and characterization of InAs/InGaAs sub-monolayer quantum dot solar cell with dot-in-a-well structure, Curr. Appl. Phys. 16 (2016) 587-592. https://doi.org/10.1016/j.cap.2016.02.009.

[17] C.G. Bailey, D. V. Forbes, R.P. Raffaelle, S.M. Hubbard, Near 1 V open circuit voltage InAs/GaAs quantum dot solar cells, Appl. Phys. Lett. 98 (2011). https://doi.org/10.1063/1.3580765.

[18] S. Kondratenko, A. Yakovliev, S. Iliash, Y. Mazur, M. Ware, P. Lam, M. Tang, J. Wu, H. Liu, G. Salamo, Influence of built-in charge on photogeneration and recombination processes in InAs/GaAs quantum dot solar cells, J. Phys. D. Appl. Phys. 50 (2017). https://doi.org/10.1088/1361-6463/aa61d4. 
[19] D. Kim, M. Tang, J. Wu, S. Hatch, Y. Maidaniuk, V. Dorogan, Y.I. Mazur, G.J. Salamo, H. Liu, Si-Doped InAs/GaAs Quantum-Dot Solar Cell with AlAs Cap Layers, IEEE J. Photovoltaics. 6 (2016) 906-911. https://doi.org/10.1109/JPHOTOV.2016.2547581.

[20] F.K. Tutu, P. Lam, J. Wu, N. Miyashita, Y. Okada, K.H. Lee, N.J. Ekins-Daukes, J. Wilson, H. Liu, InAs/GaAs quantum dot solar cell with an AlAs cap layer, Appl. Phys. Lett. 102 (2013). https://doi.org/10.1063/1.4803459.

[21] H. Yokota, K. Iizuka, H. Okamoto, T. Suzuki, AlAs coating for stacked structure of selfassembled InAs/GaAs quantum dots, J. Cryst. Growth. 301-302 (2007) 825-827. https://doi.org/10.1016/j.jcrysgro.2006.11.326.

[22] A.F. Tsatsul'nikov, A.R. Kovsh, A.E. Zhukov, Y.M. Shernyakov, Y.G. Musikhin, V.M. Ustinov, N.A. Bert, P.S. Kop'ev, Z.I. Alferov, A.M. Mintairov, J.L. Merz, N.N. Ledentsov, D. Bimberg, Volmer-Weber and Stranski-Krastanov InAs-(Al,Ga)As quantum dots emitting at $1.3 \mu \mathrm{m}$, J. Appl. Phys. $88 \quad$ (2000) 6272-6275. https://doi.org/10.1063/1.1321795.

[23] A. Varghese, M. Yakimov, V. Tokranov, V. Mitin, K. Sablon, A. Sergeev, S. Oktyabrsky, Complete voltage recovery in quantum dot solar cells due to suppression of electron capture, Nanoscale. 8 (2016) 7248-7256. https://doi.org/10.1039/c5nr07774e.

[24] A. Belghachi, A. Helmaoui, Effect of the front surface field on GaAs solar cell photocurrent, Sol. Energy Mater. Sol. Cells. 92 (2008) 667-672. https://doi.org/10.1016/j.solmat.2008.02.003.

[25] P.D. Demoulin, M.S. Lundstrom, R.J. Schwartz, Back-surface field design for n+p GaAs cells, Sol. Cells. 20 (1987) 229-236. https://doi.org/10.1016/0379-6787(87)90030-5.

[26] T. Inoue, S. Kido, K. Sasayama, T. Kita, O. Wada, Impurity doping in self-assembled InAs/GaAs quantum dots by selection of growth steps, J. Appl. Phys. 108 (2010) 063524. https://doi.org/10.1063/1.3483252.

[27] D. V. Regelman, E. Dekel, D. Gershoni, E. Ehrenfreund, A.J. Williamson, J. Shumway, A. Zunger, W. V. Schoenfeld, P.M. Petroff, Optical spectroscopy of single quantum dots at tunable positive, neutral, and negative charge states, Phys. Rev. B - Condens. Matter Mater. Phys. 64 (2001) 1653011-1653017. https://doi.org/10.1103/PhysRevB.64.165301.

[28] S. M. Sze . Kwok K. Ng, Physics of Semiconducting Devices, Third, John Wiley \& Sons, Inc., Hoboken, New Jersey, United States of America, 2007.

[29] S. Suckow, 2/3-Diode Fit, (2014). https://doi.org/https://nanohub.org/resources/14300.

[30] H.F. Lu, L. Fu, G. Jolley, H.H. Tan, S.R. Tatavarti, C. Jagadish, Temperature dependence of dark current properties of InGaAs/GaAs quantum dot solar cells, Appl. Phys. Lett. 98 (2011) 183509. https://doi.org/10.1063/1.3586251. 
[31] M.C. Löbl, S. Scholz, I. Söllner, J. Ritzmann, T. Denneulin, A. Kovács, B.E. Kardynał, A.D. Wieck, A. Ludwig, R.J. Warburton, Excitons in InGaAs quantum dots without electron wetting layer states, Commun. Phys. 2 (2019) 1-7. https://doi.org/10.1038/s42005-019-0194-9.

[32] nextnano - Software for semiconductor nanodevices, (n.d.).

[33] S. Golovynskyi, O. Datsenko, L. Seravalli, O. Kozak, G. Trevisi, P. Frigeri, I.S. Babichuk, I. Golovynska, J. Qu, Deep levels in metamorphic InAs/InGaAs quantum dot structures with different composition of the embedding layers, Semicond. Sci. Technol. 32 (2017). https://doi.org/10.1088/1361-6641/aa91e7.

[34] O.V. Vakulenko, S.L. Golovynskyi, S.V. Kondratenko, Effect of carrier capture by deep levels on lateral photoconductivity of InGaAsGaAs quantum dot structures, J. Appl. Phys. 110 (2011). https://doi.org/10.1063/1.3626051.

[35] S.V. Kondratenko, O.V. Vakulenko, Y.I. Mazur, V.G. Dorogan, E. Marega, M. Benamara, M.E. Ware, G.J. Salamo, Deep level centers and their role in photoconductivity transients of InGaAs/GaAs quantum dot chains, J. Appl. Phys. 116 (2014). https://doi.org/10.1063/1.4902311.

[36] T. Laine, K. Saarinen, J. Mäkinen, P. Hautojärvi, C. Corbel, L. Pfeiffer, P. Citrin, Observation of compensating Ga vacancies in highly Si-doped GaAs, Phys. Rev. B Condens. Matter Mater. Phys. 54 (1996) R11050-R11053. https://doi.org/10.1103/PhysRevB.54.R11050.

[37] M. Levinson, C.D. Coombs, J.A. Kafalas, Stability of the EL2 center in GaAs under electron-hole recombination conditions, Phys. Rev. B. 34 (1986) 4358-4359. https://doi.org/10.1103/PhysRevB.34.4358. 